\title{
Ions Passage Through Nanodroplets in a Multicomponent Beam
}

\author{
I. Hasanov*, I. Gurbanov And E. Akbarov \\ Institute of Physics of ANAS, G. Javid av., 131, Az1143 Baku, Azerbaijan
}

\begin{abstract}
The report is a part of researches of the characteristics of nanodisperse phase of liquid metal ion source. In the certain mode of the liquid metal source the ions and the nanodroplets are simultaneously generated. Fast ions (In, $\mathrm{Sn}, \mathrm{Au}, \mathrm{Ge}$ ) are passing through droplets and are losing a part of energy at collisions. Generation of nanodroplets is accompanied by excitation of capillary waves on the surface of the liquid emitter which results in high-frequency modulation of the ion current. In the experiments, droplets with the sizes of $2-20 \mathrm{~nm}$ and a characteristic specific charge of $5 \times 10^{4} \mathrm{C} / \mathrm{kg}$ were registered. Energy spectra of ions were defined by means of the filter of speeds (Vine's filter) with crossed static electromagnetic fields. The reduction of energy of In+ ions in near-axis areas of the beam achieves $250 \mathrm{eV}$ at acceleration voltage of $6 \mathrm{kV}$ in the conditions of our measurements. Outside of this area there are no losses of energy, as the nanodroplets extend along the beam axis. Penetration depth of the accelerated ions in liquid indium is estimated within the framework of the model of Linhard-Scharff-Schiott, considering interaction of ions with nuclei of the target. A similar interaction between components has to be considered in ion-beam systems with complex composition where there is a relative movement of the various charged particles. Nanodroplet deposition on a conductive surface is of interest for creation of various nanostructures. By having a substrate at a distances of tens microns from the needle and by moving it with respect to the needle by means of piezostage, indium stripes of micron width were deposited.
\end{abstract}

DOI: 10.12693/APhysPolA.134.119

PACS/topics: ion source, ion beam, energy spectra, nanoparticle

\section{Introduction}

Power losses of the accelerated ions occur, for example, at interaction of beams with the surface of a solid state. At movement of the accelerated ions through the condensed substance their non-elastic collisions with electrons and elastic collisions with nuclei of atoms [1] are considered. At low energy of ions their penetration into substance, up to a full stop, is mainly determined by interactions with nuclei.

Evolution of the distribution of ions occurs also inside beams with high concentration of particles. Under action of fluctuating electric fields the cross-sectional energy spread of ions grows and as a consequence, the brightness of beams decreases [2]. Coulomb collisions between negative ions of hydrogen result in loss of a part of the beam current [3].

Collisions can occur also between various fragments of the beam at their relative movement. In the present work power losses of ions in liquid metal source are considered. Under certain conditions ions and charged nanodroplets are generated in such sources. Deposition of these ions and charged nanodroplets on a solid surface is of interest for formation of various quantum structures.

\section{Experiment}

For creation of beams a compact ion source of container type [4] was used (Fig. 1). The working substance (Sn, $\mathrm{In}, \mathrm{Au}, \mathrm{Ge}, \mathrm{BNiAl})$ with the moistened needle (W, Fe,

\footnotetext{
*corresponding author; e-mail: ilkhamg@yahoo.com
}

$\mathrm{Ni}$ ) was located in graphite container which was heated up from the back side by electron bombardment up to fusion temperature of the working substance.
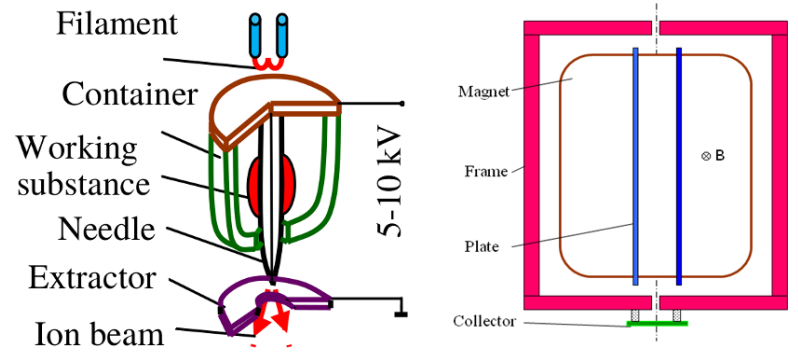

Fig. 1. The scheme of experiments with liquid metal source and the filter of ion velocities.

Voltage accelerating the ions was measured directly between the needle and the extractor, i.e. after the limiting resistance. The beam composition and spectra of energy of the ions were defined by means of the analyzer with crossed $E \perp B$ fields (similar to the Vine's filter of speeds). The mass analyzer could be moved with respect to the axis of the beam along two dimensions by means of micrometric screws without infringement of vacuum.

Spectra of oscillations of the beam current were registered by S4-25 analyzer with frequency range up to $60 \mathrm{MHz}$. Distribution of the ion current density along the beam radius was defined with the help of a multichannel compact probe. All measuring systems were calibrated with the help of standard devices, and physical measurements were repeated for accumulation of the statistical data. The experimental system was mounted on the basis of vacuum setup, A700-Q Leybold-Heraeus, with limiting vacuum of $5 \times 10^{-6}$ Torr. 


\section{Results and discussion}

It is known, that at small ion currents the emission of liquid metal sources is stable. At a certain threshold current (of about $40 \mu \mathrm{A}$ ) the high-frequency oscillations are excited in the beam, which are accompanied by the generation of charged nanodroplets with the sizes of 2-20 nm and a specific charge $q / m=5 \times 10^{4} \mathrm{C} / \mathrm{kg}(\mathrm{In}, \mathrm{Sn})[5,6]$.

The size distribution of the nanodroplets is described by a decreasing exponential function. The quantity of particles of the smallest size exceeds the number of particles of the largest size by three orders of magnitude. Estimations show that on the average for every 16 atoms of a nanodroplet one elementary charge is necessary.

Oscillations of the beam current are caused by development of capillary instability of the Taylor cone on the surface of which a system of standing waves is established. The discrete form of the spectrum of the oscillations is caused by these standing waves. A picture of emission of the charged particles from the described source is presented schematically in Fig. 2.

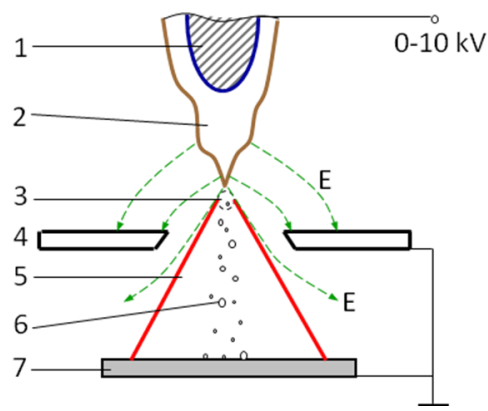

Fig. 2. Emission of ions and nanodroplets: 1 needle, 2 liquid Taylor cone, 3 lighting zone, 4 extractor, 5 ion beam, 6 nanodroplets, 7 collector.

Ions are emitted from a small spot at the top of the Taylor cone. The divergence angle of the ion beam reaches $90^{\circ}$. At a sufficient value of intensity of the electric field an abruption of nanodroplet occurs from top of the cone. Their stream has a divergence angle of 2 $3^{\circ}[7]$. In experiments it was found, that at the excitation of the emitter instability the density of ion current in the center of the beam is a little reduced in comparison with its periphery.

Spectra of energy of the ions, which were repeatedly reproduced at preservation of experimental conditions, were registered in the center of the beam (Fig. 3a) and outside of its axis (Fig. 3b) [8]. The last was obtained by moving the mass analyzer across the axis of the beam. Spectra were obtained in two modes: in absence of nanodroplet generation (curve $1, I_{\mathrm{b}}=30 \mu \mathrm{A}, U_{\mathrm{b}}=6 \mathrm{kV}$ ) and at their generation (curve $2, I_{\mathrm{b}}=50 \mu \mathrm{A}, U_{\mathrm{b}}=6.2 \mathrm{kV}$ ).

To ions with energy of $6 \mathrm{keV}$ corresponds the intensity of electric field in the analyzer of $140.5 \mathrm{~V} / \mathrm{cm}$. It is necessary to note, that the position of the entrance slit of the analyzer was adjusted with respect to the axis of a beam in such way that the maximum of passing current was reached at a potential difference between the plates

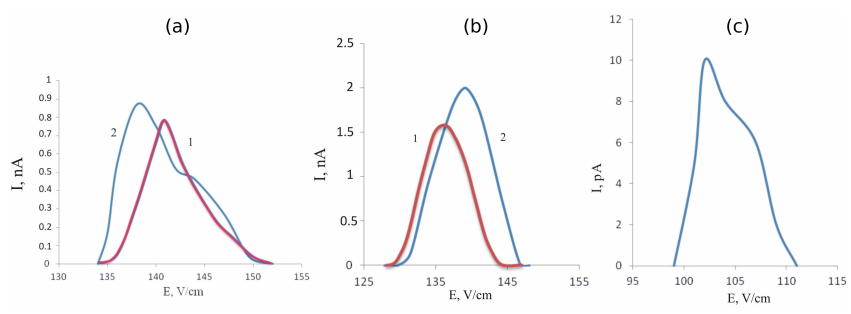

Fig. 3. Ion current through the filter of velocities as function an electric field intensity for $\operatorname{In}_{1}^{+}$(curve $1: I_{\mathrm{b}}=$ $30 \mu \mathrm{A}, U=6 \mathrm{kV}$; curve $2 I_{\mathrm{b}}=50 \mu \mathrm{A}, U=6.2 \mathrm{kV}$ ) (a) in the beam center, and (b) outside the beam axis. Ion current for $\operatorname{In}_{2}^{+}: I_{\mathrm{b}}=50 \mu \mathrm{A}, U=6.2 \mathrm{kV}$, in the beam center (c).

equal to the calculated value. The position of the analyzer was not further varied at registration of the charged nanodroplets. The spectrum of energy of double-atom ions $\mathrm{In}_{2}^{+}$was also registered in the mode of nanodroplet generation (Fig. 3c).

In Fig. 3, at nanodroplet generation the maximum of spectrum in the center of the beam shifts towards smaller energy by $250 \mathrm{eV}$, however outside beam axis it shifts towards greater energy. The last shows that for the increase of the current of the beam an increase of extraction voltage $U_{\mathrm{b}}$ is necessary.

As was specified above, the charged nanoparticles in liquid metal sources propagate in the form of weekly divergent beam with an angle of expansion of $2-3^{\circ}$. Absence of the delay of the ions moving outside of beam axis (Fig. 3b) testifies that the reduction of energy of ions occurs owing to their interaction with charged nanodroplets at propagation along the system axis. In conditions of our experiments the relative speed of ions and nanodroplets is equal to $0.75 v_{\mathrm{i}}$, where $v_{\mathrm{i}}$ is the speed of ions reached in the accelerating field.

Interaction of ions with nanodroplets occurs during the flight from the needle up to the entrance into the mass analyzer (distance of about $10 \mathrm{~mm}$ ). Further the magnetic field of the analyzer divides the various components of the beam.

The run of the accelerated ions in the condensed substance is considered in the theory of Coulomb collisions with electrons and nuclei of atoms [1]. At low energies of ions their braking occurs as the result of elastic interaction with nuclei, and the run in substance is estimated from the Eq. (1).

$$
R_{\mathrm{n}}=2 k E_{0}, k=\frac{1.8\left(Z_{1}^{2 / 3}+Z_{2}^{2 / 3}\right)^{1 / 2}}{N Z_{1} Z_{2}} \frac{M_{1}+M_{2}}{M_{2}} .
$$

Here $k$ is measured in $\mathrm{nm} / \mathrm{eV}, Z_{1}, M_{1}$ and $Z_{2}, M_{2}$ are atom number and mass of the primary ion and of the atoms of substance, respectively, $N$ is concentration of atoms, $\mathrm{nm}^{-3}$.

At $E_{0}=3.38 \mathrm{keV}$, corresponding to relative speed of ion and nanoparticle $v_{\mathrm{i}}-v_{\mathrm{np}}$, and $N=34.1 \mathrm{~nm}^{-3}$ for liquid indium, $\mathrm{Eq}(1)$ gives value of $R_{\mathrm{n}}=1.54 \mathrm{~nm}$. Run of the ions is smaller than the registered size 
$d_{\mathrm{np}}=2 \mathrm{~nm}$ of the majority of nanodroplets. That means that ions should lose all energy and should not pass through the nanoparticles.

However, it is necessary to take into account that the probability of flight of an ion along the diameter of a droplet is much smaller than that along the thinner parts. Apparently, in liquid metal source the charged nanodroplets and clusters with the sizes smaller than the calculated run of ions $R_{\mathrm{n}}=1.54 \mathrm{~nm}$ are intensively generated [9].

For registration of such small particles the resolution of used Tesla electron microscope was insufficient. Ions lose a part of their energy at passage through small nanodroplets and leave them having a similar direction.

The charge condition of the fast ion passing through a nanodroplet, demands a separate consideration. There are experiments in which the stream of fast neutral atoms propagating along the beam axis was registered [10]. It is allowable, that a part of near axis ions was neutralized during the flight through small nanodroplets in our experiments.

Apparently in Fig. 3a (curve 2), there is a part of ions which have rather high energy. Most likely, this group of ions was propagating outside the nanoparticle beam and did not interact with them. A similar form of peak in spectrum of energy is observed for doubly-ionized ions (Fig. 3c), that testifies about their participation in the specified processes alongside the singly-ionized ions.

Absorption of a part of near axis ions and loss of charge by them as a result of collisions with nanodroplets also shows the reduction of current density in the center of the beam.

Considering small divergence of the nanodroplet stream, the deposition of narrow conducting stripes is of interest at perpendicular movement of a sample. For this purpose, it is possible to not use complex ion optics, and to approach the source needle to the surface of a sample as much as possible (Fig. 4a).

Thus, it is necessary to take into account that because of high ion current density it is probable to overheat the surface. For precision movement of a sample a piezo stage MS 2000 was used, which was placed in the vacuum chamber. In the preliminary experiments indium stripes with a width of about micron and a length of some millimeters were deposited on the polished tungsten surface (Fig. 4b). Substantial heating the substrate was observed during deposition. In spite of the fact that in the spectrum of nanodroplets the particles with the sizes of $2 \mathrm{~nm}$ prevail, in the deposited strip the grains have sizes of 100 200nm. Apparently, in conditions of high nanodroplet concentration there was their fast conglomeration that could determine the minimal width of the stripes.

\section{Conclusions}

The estimations caried out within the framework of Linhard-Scharff-Schiott model allow assuming that in liquid metal source there is a penetration of fast ions into heavy charged nanoparticles. It results in appreciable
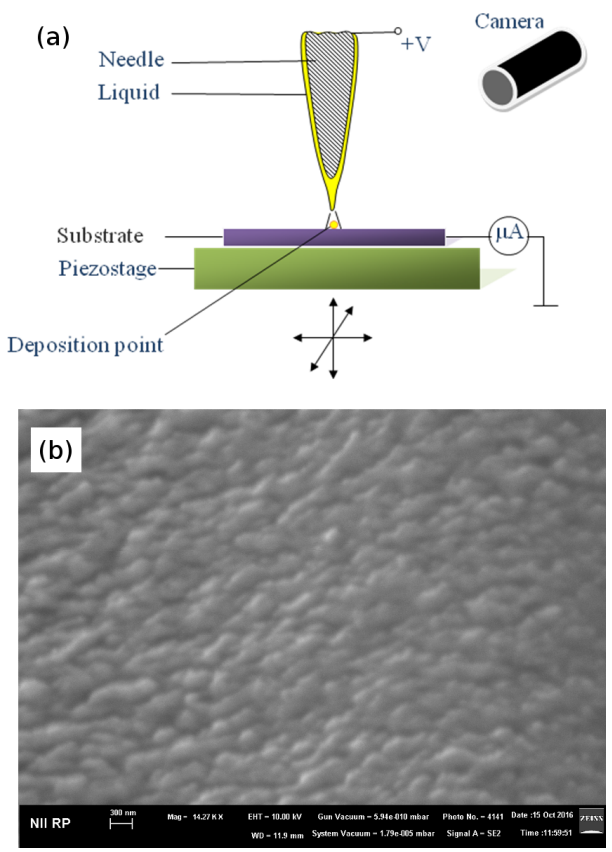

Fig. 4. Scheme of nanodroplet deposition without ion optics (a) and structure of the deposited stripe (b).

loss of energy of ions (nearly $250 \mathrm{eV}$ at full energy of beam of $6 \mathrm{keV}$ ). Similar processes are necessary to take into account at precision focusing the ion beams, at separation of ions in energy- and mass-analyzers. Registration of spectra by means of the energy analyzers is necessary for correct quantitative estimations. Registration of the size of nanoparticles by means of an electron microscope of high resolution is also required. For deposition of thinner stripes it is necessary to approach the needle of the source to a sample and to provide heat removal from the heated area. In this case a fast hardening of nanodroplets would take place on the cooled surface without their fusion.

\section{References}

[1] I. Brodie, J. Muray, The Physics of Microfabrication, Plenum Press, New York, London 1982.

[2] V. Dudnikov, A. Shabalin, J. Teck. Phys. 60, 131 (1990).

[3] A. Shishlo, J. Galambos, A. Aleksandrov, V. Lebedev, M. Plum, Phys. Rev. Lett. 108, 114801 (2012).

[4] I. Gasanov, I. Gurbanov, Jap. J. Appl. Phys. 47, 8226 (2008).

[5] I. Gasanov, I. Gurbanov, Rev. Sci. Inst. 83, $02 \mathrm{~B} 906$ (2012).

[6] A. Jaworek, J. Mater. Sci. 42, 266 (2007).

[7] Charged Particle Optics, edited by J. Orloff, CRC Press, London, New York 2009.

[8] I. Gasanov, I. Gurbanov, E. Akbarov, Eur. Phys. J. D 69, 75 (2015).

[9] D. Barr, J. Vac. Sci. Technol. B 5, 184 (1987).

[10] S.P. Thompson, Vacuum 34, 223 (1984). 